\title{
A Challenge to Microscopists
}

\section{Alwyn Eades}

Professor of Materials Science and Engineering Emeritus, Lehigh University, Lehigh University, Bethlehem, PA 18015

jae5@lehigh.edu

A wonderful thing Wikipedia

Acknowledged by all of the media

Everything's in it

From "epoch" to "minute"

And a number of bits that are seedier [1]

Wikipedia is indeed a splendid achievement. It is the place we all go on an almost daily basis to find out about things. It is also the place where other people first make contact with the topic of microscopy. Prompted by this thought, I have searched Wikipedia for a number of things related to microscopy-and our field comes up sadly lacking. The Microscopy Society of America (MSA) has an entry with a paltry four lines and does not describe much of the good work that MSA does; International Federation of Societies for Microscopy (IFSM) has no entry at all; Electron microscope has a rather uneven entry; EDS has an entry which is (in my view) unclear and out of date; the entry for Secondary electrons is two short, unhelpful paragraphs. And so on.

As microscopists we need to show a better face to the world. We need to get the Wikipedia entries in our field to be accurate, up-to-the-minute, attractive, and helpful. So my challenge to each of you is this: contribute to making the entries related to microscopy in Wikipedia much, much better. For those of you who have not done so, it is very easy to make contributions and changes to Wikipedia. Anyone can do it. If someone as inept at things computational as I am can do it (and I have made contributions to Wikipedia), be assured that you can.

I have a strong view about Wikipedia entries in technical fields. The information should be presented at multiple levels. There should be an initial part that explains the topic in terms that the layperson can understand. Think of explaining it to a middle-school child, for example. Then technical details at increasing levels of sophistication may follow. An entry that jumps in at the state of the art is not helpful (see, for example, the entry on Abbe sine condition). Articles in mainstream physics seem to try to follow the "begin simple then elaborate" model, with varying levels of success. See for example: Gravity, Force, or Acceleration.

There are two ways the microscopy community could approach this. MSA could form a group of people (a sub-committee of the Educational Outreach Committee, for example) who would take on the job of making microscopy-related Wikipedia pages better. However, that seems to go somewhat against the spirit of Wikipedia, which is that individuals contribute, both in setting up pages and in improving pages that already exist. Thus it is a dynamic and evolving process. So my challenge to each of you is this: please go to Wikipedia and look up the pages for several topics related to microscopy. If a page does not exist for a topic that interests you, create that page. If existing pages can be improved, set to work and improve them.

Let's do a better job of showing off our achievements.

[1] I plan to add this limerick to the Wikipedia page on limericks. So if you have suggestions for improvement or a better limerick in praise of Wikipedia that is quite different, please send it to me at jae5@lehigh.edu. The editor tells me that he will offer a prize for the best submission.

\section{Expand your Knowledge of Microscopy with}

\section{Whether your primary focus is
in the biological or the physica sciences, MSA takes your knowledge to the next level!}

Members Receive:

- A personal subscription to MSA's official journal, Microscopy and Microanalysis, and MSA's popular bi-monthly magazine, Microscopy Today.

- Peer Networking through the Society's Focused Interest Groups and Local Affiliated Societies.

- MSA Awards Programs, Scholarships, Speaker Opportunities, and much more!

Join MSA Today!

For more information: visit www.microscopy.org

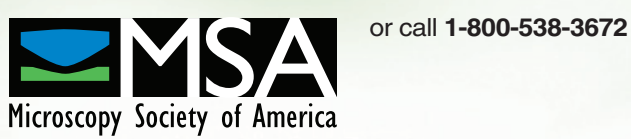




\section{Growing the Future of Microscopy}

Krista Wilks of the Chehalis School District shapes the future of young adults by teaching the basics of electron microscopy to her high school students. She encourages them to love and learn more about science through the use of electron microscopy.

Krista's choice instrument to grow future scientists? The TESCAN VEGA SEM.

Now is the time to explore nanospace with TESCAN. www.tescan-usa.com

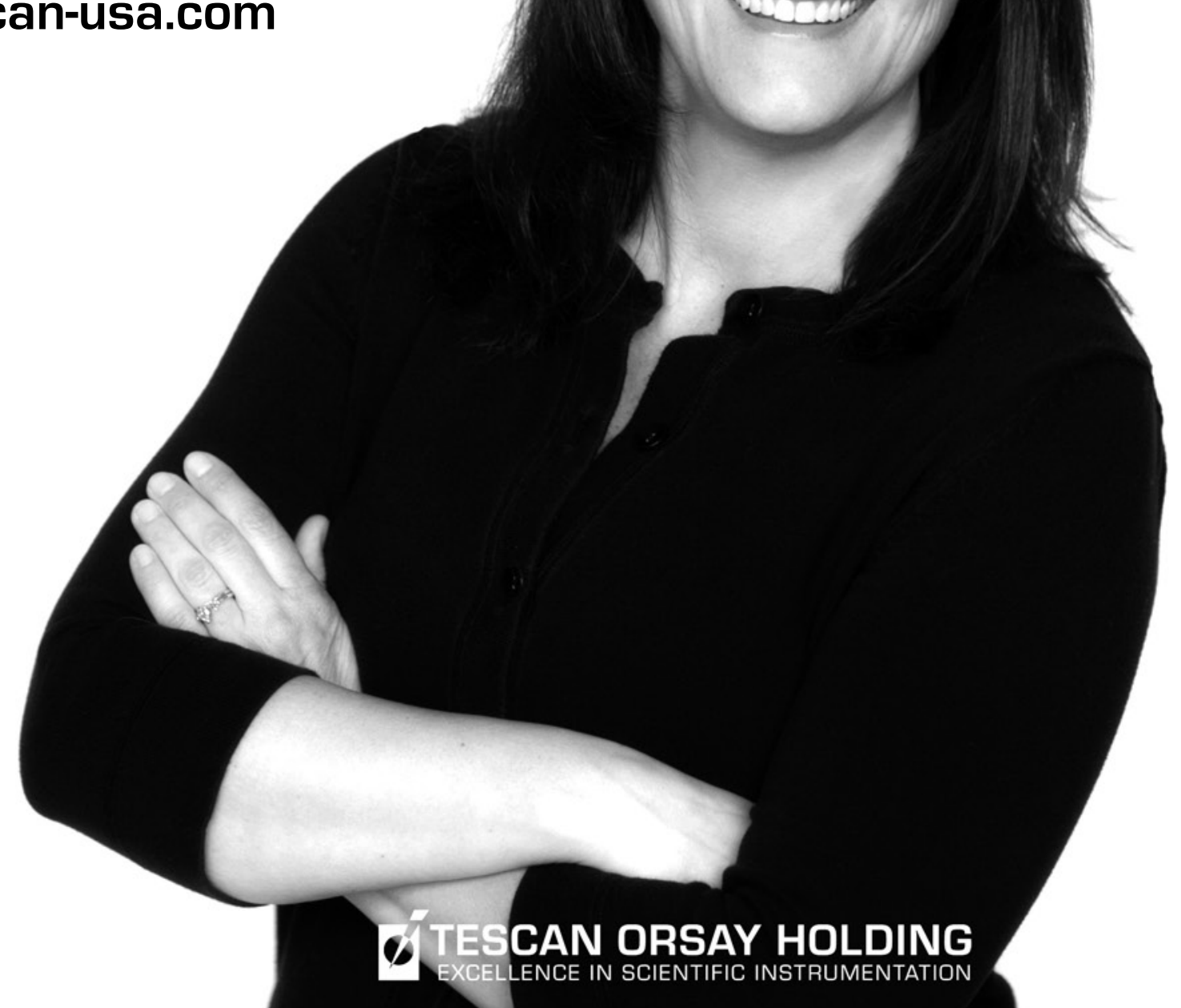

